\title{
Clima de Ambiente Acadêmico nos Grupos dos WhatsApp de Sala de Aula de Graduandos
}

\author{
Emanuel Rodrigues do Nascimento - CIN/UFPE - emanuelrdn@ hotmail.com - \\ https://orcid.org/0000-0001-6822-5407 \\ Patrícia Cabral de Azevedo Restelli Tedesco - CIN/UFPE - pcart@ cin.ufpe.br - \\ https://orcid.org/0000-0001-9450-9219
}

Resumo: Este artigo tem como objetivo aplicar os estudos sobre o clima de ambiente acadêmico nos grupos do WhatsApp de salas de aula de graduandos e diagnosticar os impactos nas atividades acadêmicas. Para isso, foi realizado um estudo de caso, com graduandos das instituições de ensino superior, no município de Caruaru-PE. Além disso, foram realizados: uma pesquisa bibliográfica, contemplando 22 estudos; levantamento de dados em campo, oriundos de 2.401 questionários aplicados; e um processo estatístico, a fim de confirmar o clima e seus efeitos. Os resultados confirmaram a presença do clima de ambiente acadêmico nos grupos do WhatsApp de sala de aula e sua influência nos comportamentos dos graduandos, por consequência nas suas atividades acadêmicas.

Palavras-chaves: WhatsApp, clima de ambiente acadêmico, teoria de resposta ao item.

\section{Academic Environment Climate in Groups of WhatsApp of Classroom of Graduates}

Abstract: This article aims to apply studies on the climate of the academic environment in WhatsApp groups in classrooms of the graduates and diagnose the impacts on academic activities. For that, a case study was carried out, with graduates from higher education institutions, in the municipality of Caruaru-PE. Furthermore, were done: a bibliographic search, contemplating 22 studies; field data collection, arising from 2.401 questionnaires applied; and a statistical process, in order to confirm the climate and its effects. The results confirmed the presence of the climate of the academic environment in the classroom WhatsApp groups and its influence on undergraduate behaviors, for consequences in their academic activities.

Keywords: WhatsApp, academic environment climate, item response theory.

\section{INTRODUÇÃO}

Os estudos sobre clima da sala de aula têm demonstrado que a forma como os discentes percebem a "atmosfera" no ambiente influencia, significativamente, os seus comportamentos, por consequência suas atividades acadêmicas (SCHWEIG; HAMILTON; BAKER, 2009; BRITO; COSTA, 2010; MORO et al., 2017). É importante ressaltar que há vários climas atuando na sala de aula, destaque, o clima de ambiente acadêmico. Esse clima, quando positivo, pode influenciar no nível de expectativa de aprendizagem dos discentes, na absorção dos conteúdos da aula, nas trocas de conhecimentos e experiências, e etc. (SCHWEIG; HAMILTON; BAKER, 2009).

Também é importante trazer na discussão que, na atualidade, esta sala de aula não mais se restringe ao ambiente físico, desde que foi introduzido o aplicativo WhatsApp na vida acadêmica dos discentes, principalmente dos graduandos. Para Lopes e Vas (2016), o uso do aplicativo WhatsApp na função de formar grupos de sala de aula, faz desse ambiente uma extensão da sala de aula. Embora não existam estudos sobre o clima de ambiente acadêmico atuando nos grupos do WhatsApp de sala de aula. Portanto, este estudo tem como pergunta condutora: Como a aplicação dos estudos do clima de ambiente acadêmico, nos grupos do WhatsApp de salas de aula de graduandos, pode contribuir para diagnosticar os impactos nas atividades acadêmicas? 
Para solucionar este questionamento, foi realizado um estudo de caso, com graduandos das instituições de ensino superior, no município de Caruaru-PE. Cabe salientar que nessa localidade há várias instituições de ensino superior. Considerando esse contexto, o presente estudo tem como objetivo: aplicar os estudos sobre clima de ambiente acadêmico nos grupos de salas de aula de graduandos e diagnosticar os impactos nas atividades acadêmicas.

Este estudo tem como benefício aplicar, de forma pioneira, os estudos do clima de sala de aula nos grupos do WhatsApp: isto produziu informações necessárias aos envolvidos na educação para poder identificar a influência do clima de ambiente acadêmico nas ações dos graduandos. Essas informações, também, servirão para corrigir o clima ou potencializá-lo, dependendo dos seus efeitos.

Para o desenvolvimento deste estudo, foram utilizadas pesquisas bibliográficas e levantamento de dados em campo, além do processo estatístico. A pesquisa bibliográfica fundamentou-se em 22 estudos; o levantamento dos dados em campo e o processo estatístico estão detalhados na seção da metodologia. É de fundamental importância destacar que a influência do clima foi estimada através da Teoria de Resposta ao Item (TRI). Segundo Pasquali e Primi (2003) a TRI funciona como se fosse um experimento, pois através de um estímulo comportamental ou construtos (variáveis não observáveis) clima num ambiente - os comportamentos no presente ou futuro dos indivíduos são influenciados, isto é, são influenciados em função do seu nível de traço latente.

\section{METODOLOGIA}

\subsection{Levantamento dos dados em campo}

No período de 22 de agosto de 2019 a 11 de novembro de 2019, foram aplicados 2.401 questionários para graduandos de 9 instituições de ensino superior (3 públicas, 3 privadas presenciais e 3 privadas semipresenciais), em Caruaru-PE. Nas instituições públicas foram alocados $34 \%$ dos questionários aplicados e nas instituições privadas presenciais e semipresenciais foram alocados $33 \%$ cada uma.

Dentro dessas instituições, os graduandos foram abordados de forma individual e aleatória. As suas participações na pesquisa estavam condicionadas a uma data de referência, 21 de agosto de 2019, e a estes critérios: ser maior de idade; ter realizado pelo menos uma prova e uma atividade extraclasse; ter participado de pelo menos um grupo do WhatsApp de sala de aula, turmas ou disciplinas. De fato, isso foi de fundamental importância para mensurar o clima do ambiente acadêmico e seus efeitos, pois a partir do cumprimento desses requisitos, os graduandos estavam submetidos a esse clima, logo os seus comportamentos foram influenciados ao longo do tempo. Dessa forma, a data de referência representou o limite dessa influência e a coleta de dados capturou esse fenômeno.

Essa coleta foi aprovada por três comitês de ética em pesquisa e também recebeu parecer favorável em sua conclusão (CAAE: 15399319.6.0000.5208; 15399319.6.3009.5191 e 15399319.6.3003.5203).

\subsection{Procedimento estatístico}

Há dois pressupostos fundamentais para estimar o modelo unidimensional da TRI para dados dicotômicos: o primeiro, é a independência local, que presume não haver condicionamento entre os itens no questionário (sem interferência) e o segundo é a unidimensionalidade, que presume que as respostas aos itens são influenciadas, exclusivamente, pelo tamanho de um teta dominante (traço latente) do sujeito 
(PASQUALI; PRIMI, 2003; PASQUALI, 2007).

Observando esses pressupostos e os métodos na literatura para estimar o modelo mais ajustado, foram traçadas 5 etapas, destalhadas no quadro 1: a etapa 1 concentrou-se na adequação dos dados - o questionário é composto por 82 itens e após uma análise crítica do primeiro pressuposto e os itens de aspectos pessoais, então foram excluídos 32 itens, deste modo, restaram apenas 38 itens dicotômicos e 12 itens politômicos, sendo que este foi dicotomizado -. É importante ressaltar que dicotomizar dados é uma prática comum na literatura, como no trabalho de Maydeu-Olivares, Hernandez e Mcdonald (2006).

Quadro 1 - Etapas para estimar o modelo

\begin{tabular}{|c|c|c|c|}
\hline Etapas & Técnica & Objetivo & Recomendação \\
\hline $\begin{array}{l}1^{\circ} \text { Adequação } \\
\text { dos dados }\end{array}$ & Dicotomizar & $\begin{array}{l}\text { Padronizar o questionário com } \\
\text { dados dicotômicos, para utilizar o } \\
\text { modelo da TRI. }\end{array}$ & $\begin{array}{l}\text { Atribui-se "0" para itens com } \\
\text { ausência de comportamentos e } \\
\text { "1" a itens com presença. (1) }\end{array}$ \\
\hline \multirow{4}{*}{$\begin{array}{l}2^{\circ} \text { Requisitos } \\
\text { para realizar } \\
\text { uma análise } \\
\text { fatorial }(\mathrm{AF})\end{array}$} & $\begin{array}{l}\text { Análise do } \\
\text { Tamanho da } \\
\text { Amostra } \\
\end{array}$ & $\begin{array}{l}\text { Averiguar se o tamanho da amostra } \\
\text { é ideal para realizar uma análise } \\
\text { fatorial. }\end{array}$ & $\begin{array}{l}\text { Para cada item são necessários } \\
10 \text { indivíduos e o mínimo total } \\
\text { de } 100 \text { indivíduos. (2) }\end{array}$ \\
\hline & $\begin{array}{l}\text { Correlação } \\
\text { Tetracórica }\end{array}$ & $\begin{array}{l}\text { Analisar se os itens dicotômicos } \\
\text { podem associar-se com algum fator } \\
\text { (dimensão do construto). }\end{array}$ & $\begin{array}{l}\text { A maioria das correlações na } \\
\text { matriz deve ser } \geq 0,3 \text {. (3) }\end{array}$ \\
\hline & $\begin{array}{c}\text { Teste da } \\
\text { Esfericidade } \\
\text { de Bartlett } \\
\text { (BTS) }\end{array}$ & $\begin{array}{l}\text { Testar a hipótese, se os itens se } \\
\text { agrupam-se para formar dimensões. }\end{array}$ & $\begin{array}{l}\text { Testar Hipótese: } \\
H_{0}: \text { a matriz de correlação é } \\
\text { uma matriz identidade. } H_{1}: \text { a } \\
\text { matriz de correlação não é uma } \\
\text { matriz identidade. Isso significa } \\
\text { para } H_{0} \text { que os itens não se } \\
\text { agrupam para formar nenhum } \\
\text { fator e } H_{1} \text { forma pelo menos um } \\
\text { fator. Rejeita-se } H_{0} \text {, quando } \\
P<0,05 \text {. (3) }\end{array}$ \\
\hline & $\begin{array}{l}\text { Teste de } \\
\text { Kaiser- } \\
\text { Meyer- } \\
\text { Olkim } \\
\text { (KMO) }\end{array}$ & $\begin{array}{l}\text { Testar a proporção da variância dos } \\
\text { itens que pode ser considerada } \\
\text { comum a todos os itens, isto é, se os } \\
\text { dados estão ajustados para AF. } \\
\text { Obs. há o geral e o individual. }\end{array}$ & $\begin{array}{l}\text { KMO: } \\
\text { Aceitável: } \geq 0,5 .(2)\end{array}$ \\
\hline \multirow{4}{*}{$\begin{array}{c}3^{\circ} \\
\text { Análise } \\
\text { Fatorial ou } \\
\text { Componente } \\
\text { Principal } \\
\text { (CP) }\end{array}$} & $\begin{array}{l}\text { Critério de } \\
\text { Guttman- } \\
\text { Kaiser }\end{array}$ & \multirow{3}{*}{$\begin{array}{l}\text { Extrair os números de dimensões da } \\
\text { AF ou CP. } \\
\text { Obs. Nas técnicas utiliza-se a } \\
\text { correlação tetracórica. }\end{array}$} & $\begin{array}{l}\text { Considerar na contagem os } \\
\text { autovalores } \\
\text { ( } 4 \text { eigenvalue })>1 .\end{array}$ \\
\hline & $\begin{array}{l}\text { Scree de } \\
\text { Cattell }\end{array}$ & & $\begin{array}{l}\text { Considerar na contagem os } \\
\text { autovalores que formarem uma } \\
\text { linha reta (esquerda para direita } \\
\text { no gráfico) ou até sofrer uma } \\
\text { queda abrupta (cotovelo) (6) }\end{array}$ \\
\hline & $\begin{array}{l}\text { Análise da } \\
\text { Paralela }\end{array}$ & & $\begin{array}{lr}\text { Considerar na } & \text { contagem os } \\
\text { autovalores } & \text { obtidos } \\
\text { empiricamente } & \text { (Actual data) } \\
\text { acima dos autovalores } \\
\text { provenientes das simulações } \\
\text { (simulated data) ou da solução } \\
\text { ótima (Resampled data). (7) }\end{array}$ \\
\hline & $\begin{array}{l}\text { Rotação } \\
\text { de Fatores }\end{array}$ & $\begin{array}{l}\text { Obter as cargas } \mathrm{AF} \text { ou } \mathrm{CP} \text { das } \\
\text { dimensões do construto. } \\
\text { Obs. Utilizou-se a correlação } \\
\text { tetracórica, rotação Varimax e o } \\
\text { método fatorial da máxima } \\
\text { verossimilhança. }\end{array}$ & $\begin{array}{l}\text { Para amostra } \geq 350 \text { indivíduos, } \\
\text { considerar na permanência da } \\
\text { dimensão as cargas } \geq 0,3 \text {. (2). }\end{array}$ \\
\hline
\end{tabular}


Cont. Quadro 1

\begin{tabular}{|c|c|c|c|}
\hline \multirow[t]{2}{*}{$\begin{array}{c}\mathbf{4}^{\mathbf{o}} \\
\text { Consistência } \\
\text { Interna }\end{array}$} & $\begin{array}{l}\text { Kuder- } \\
\text { Richardson } \\
\quad(\mathrm{KR})\end{array}$ & $\begin{array}{l}\text { Obter confiabilidade do } \\
\text { fator, isto é, se o conjunto dos } \\
\text { itens de fato mede a mesma } \\
\text { dimensão do construto e não } \\
\text { outra. }\end{array}$ & $\begin{array}{l}\text { KR: } \\
\text { Valor aceitável: } \geq 0,6 \\
\text { Valor ideal: } \geq 0,7 .(8)\end{array}$ \\
\hline & $\begin{array}{l}\text { Correlação } \\
\text { Item-Total }\end{array}$ & $\begin{array}{l}\text { Obter a contribuição de cada } \\
\text { item na consistência interna } \\
\text { da dimensão estimada. }\end{array}$ & $\begin{array}{l}\text { Correlação Item-Total: } \\
\text { Ideal: } \geq 0,3 \text {. (9) }\end{array}$ \\
\hline \multirow{6}{*}{$\begin{array}{c}5^{\circ} \\
\text { Teoria de } \\
\text { Resposta ao } \\
\text { Item }\end{array}$} & Interação & \multirow{2}{*}{$\begin{array}{l}\text { Obter o melhor modelo entre } \\
\text { 2PL, 3PL, 3PLu e 4PL } \\
\text { (modelos dicotômicos da } \\
\text { TRI). }\end{array}$} & $\begin{array}{l}\text { Observar se há convergência dos } \\
\text { parâmetros dos modelos (10) }\end{array}$ \\
\hline & $\begin{array}{l}\text { Análise da } \\
\text { Variância } \\
\text { Genérica } \\
\text { (ANOVA) }\end{array}$ & & $\begin{array}{l}\text { Comparar entre os modelos: o } \\
\text { critério de informação Akaike (AIC) } \\
\text { e Bayesiano (BIC), sendo "o menor } \\
\text { melhor" e } p \text { descritivo, sendo } H_{0} \text { : } \\
\text { modelo } n \text { ajusta bem aos dados e } H_{1} \text { : } \\
\text { modelo } n+1 \text { ajusta bem, rejeita-se } \\
H_{0} \text {, quando } p \leq 0,05 \text {. (11) }\end{array}$ \\
\hline & $\begin{array}{c}M^{2} \\
\text { (ajustamento } \\
\text { do modelo) }\end{array}$ & $\begin{array}{l}\text { Obter o ajustamento do } \\
\text { modelo. } \\
\text { Obs. O } M^{2} \text { contém: } \\
\text { Média Quadrática dos Erros } \\
\text { de Aproximação (RMSEA); } \\
\text { Raiz Padronizada da Média } \\
\text { Quadrática Residual } \\
\text { (SRMSR); Índice de Tucker- } \\
\text { Lewis (TLI); Índice de } \\
\text { Ajustamento Comparativo } \\
\text { (CFI) e o teste de hipótese. }\end{array}$ & $\begin{array}{l}\text { RMSEA: }>0,10 \text { mal ajustado; } \\
\text { ]0,08; 0,10] razoável; ]0,05; 0,08] } \\
\text { bom; } \leq 0,05 \text { muito bom. } \\
\text { SRMSR: } \geq 0,09 \text { mau ajustado; } \\
<0,09 \text { perfeito ajuste. } \\
\text { TLI e CFI: }<0,9 \text { mal ajustado; } \\
{[0,09 ; 0,95[\text { bom; } \geq 0,95 \text { muito }} \\
\text { bom. } \\
\text { Teste de hipótese: Se } p<0,05 \text {, o } \\
\text { modelo apresenta ajuste ruim, } \\
\text { embora haja erro empírico do tipo } 1 \\
\text { (rejeitar-se } H_{0} \text {, sendo verdadeiro } \\
H_{0} \text { ), principalmente quando dados } \\
\text { são dicotômicos. (2, e } 12,13,14)\end{array}$ \\
\hline & Parâmetros & \multirow{3}{*}{ 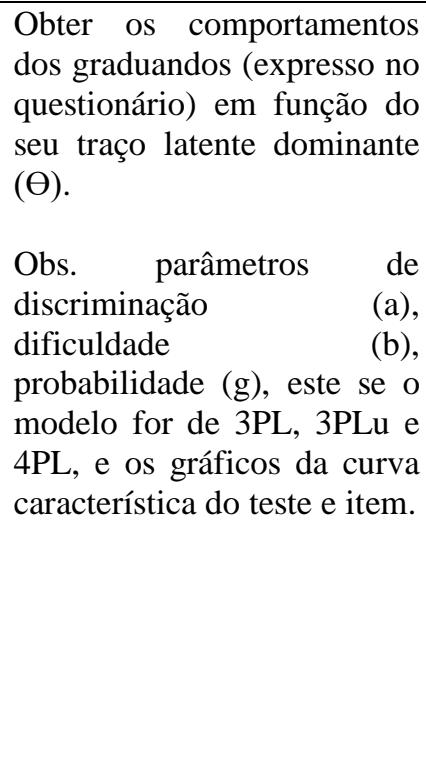 } & $\begin{array}{l}\text { Discriminação (a): } \\
=0 \text { Nenhum; ]0,01; 0,35] Muito } \\
\text { baixo; ]0,35;0,65] Baixo; } \\
\text { ]0,65; } 1,35] \text { Moderado; ]1,35; } 1,7] \\
\text { Alta; }>1,7 \text { Perfeito. } \\
\text { Dificuldade (b): } \\
\leq-3 \text { Muito fácil; ] - } 3 ;-1 \text { [ fácil; } \\
\text { [-1; } 1 \text { [ Intermediário; [1;3 [ } \\
\text { Difícil; } \geq 3 \text { Muito difícil. (15 e } 16)\end{array}$ \\
\hline & $\begin{array}{c}\text { Curva } \\
\text { Característica } \\
\text { do Teste }\end{array}$ & & $\begin{array}{l}\text { Representação gráfica da pontuação } \\
\text { (comportamento) total esperado e o } \\
\text { nível do traço latente. }(15 \text { e } 16)\end{array}$ \\
\hline & $\begin{array}{l}\text { Curva } \\
\text { Característica } \\
\text { do Item }\end{array}$ & & $\begin{array}{l}\text { Representação gráfica da } \\
\text { probabilidade de acertar o item } \\
\text { (manifestar o comportamento) e o } \\
\text { nível do traço latente. }(15 \text { e 16) }\end{array}$ \\
\hline
\end{tabular}

Fonte: 1.MAYDEU-OLIVARES, HERNANDEZ; MCDONALD, 2006; 2.HAIR JUNIOR et al, 2005; 3.FIELD; MILES; FIELD, 2012; 4.GUTTMAN, 1954; 5.KAISER, 1960; 6.CATTELL, 1966; 7.HORN, 1965; 8.KUDER; RICHARDSON, 1937; 9.STREINER; NORMAN, 2008; 10.ANDRADE; TAVARES; VALLE, 2000; 11.CHALMERS, 2012; 12.MAYDEU-OLIVARES; JOE, 2006; 13.CAI; HANSEN, 2013; 14.HU; BENTLER, 1999; 15.BAKER, 2001; 16.PASQUALI, 2007. 
Após a dicotomização dos dados, uma análise fatorial foi realizada (observandose primeiro os requisitos). Para isso, nas etapas 2, 3 e 4 utilizou-se o software $\mathrm{R} 4.0 .3 \mathrm{com}$ o pacote $p s y c h$ 2.0.9. A etapa 2 cumpriu todos requisitos para realizar uma análise fatorial. Na etapa 3, obtiveram 7 dimensões, mas pelo critério da parcimônia (menos é melhor) optou-se por 5 dimensões.

Em seguida, foi extraída a dimensão do clima de ambiente acadêmico, composto por 15 itens com cargas acima de 0,3. Na etapa 4, apesar do KR ser maior que 0,7, o critério foi atendido, mas obtiveram-se 3 itens baixo de 0,3 na correlação item-total, então estes foram excluídos, restando 12 itens. Em seguida, uma nova análise crítica foi realizada, observando as opiniões de Schweig, Hamilton e Baker (2009) sobre esse clima, descritos na introdução, resultando numa seleção de 6 itens (quadro 2).

Quadro 2 - Itens que compõem o clima de ambiente acadêmico

\begin{tabular}{|c|l|}
\hline Itens & \multicolumn{1}{|c|}{ Perguntas } \\
\hline Q01 & $\begin{array}{l}\text { Já interagiu no grupo do WhatsApp da sala de aula, durante a aula, e que a maioria dos } \\
\text { membros esteve presente na aula? }\end{array}$ \\
\hline Q02 & $\begin{array}{l}\text { Já postou algum conteúdo que encontrou na internet relacionado com a aula, no grupo do } \\
\text { WhatsApp da sala de aula, durante a aula? }\end{array}$ \\
\hline Q03 & Após a aula, já discutiu pelo grupo do WhatsApp da sala de aula os conteúdos estudados? \\
\hline Q04 & $\begin{array}{l}\text { Antes da prova, você já discutiu pelo grupo do WhatsApp da sala de aula: dúvidas dos } \\
\text { conteúdos? }\end{array}$ \\
\hline Q05 & $\begin{array}{l}\text { Após a prova, você já discutiu pelo grupo do WhatsApp da sala de aula: o conteúdo da } \\
\text { prova? }\end{array}$ \\
\hline Q06 & $\begin{array}{l}\text { Na(s) atividade(s) extraclasse, através do grupo do WhatsApp da sala de aula, você já: } \\
\text { passou Respostas? }\end{array}$ \\
\hline
\end{tabular}

Fonte: Elaboração dos autores, 2021.

Esses 6 itens foram novamente submetidos às etapas 2, 3 e 4. Na etapa 5, utilizouse o software R 4.0.3 com o pacote mirt 1.33.2. Estas etapas, são descritas na próxima seção.

\section{RESULTADOS E DISCUSSÕES}

Este estudo tem como propósito aplicar os estudos sobre clima de ambiente acadêmico nos grupos do WhatsApp de salas de aula de graduandos e diagnosticar os impactos nas atividades acadêmicas. A suposição feita a partir do problema foi que há um clima de ambiente acadêmico presente nos grupos do WhatsApp de sala de aula de graduandos que influencia seus comportamentos e, ao medir os níveis de influência, é possível diagnosticar as atividades acadêmicas nesses grupos. Isso porque, Schweig, Hamilton e Baker (2009) afirmaram que o clima de ambiente acadêmico na sala de aula influencia os comportamentos dos estudantes e, como consequência suas atividades acadêmicas. Lopes e Vas (2016) afirmaram também que os grupos do WhatsApp de sala de aula são uma extensão da sala de aula. Uma das formas de mensurar a influência desse clima é através da TRI (MORO et al, 2017).

Continuando o procedimento estatístico para estimar a TRI. Foi analisado o

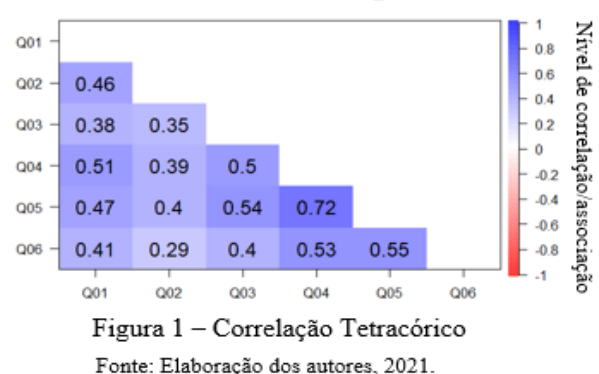
tamanho da amostra ideal em função da quantidade de itens (etapa 2). A relação dos 6 itens com amostra de 2.401 graduandos, atendeu o critério de Hair Junior et al (2005) para cada item são necessários 10 indivíduos, como um dos requisitos para realizar uma análise fatorial.

Outro requisito, é a correlação tetracórica, exposta na figura 1. A intensidade mais forte na 
cor representa uma associação mais forte entre os itens, sendo a azul de forma direta e a vermelha inversa. É observado que a maioria das associações são maiores que 0,3 , para esta dimensão, também atendendo o critério para realizar uma análise fatorial de Field, Miles e Field (2012).

Nesta etapa, ainda foram submetidos os testes BTS e KMO: aquele não aceitou o $H_{0}$ (K-quadrado de Bartlett $=47,839, \mathrm{df}=5$, valor de $\mathrm{p}=3.832 \mathrm{e}-09$ ) e aceitou $H_{1}$, onde itens agrupam-se para formar pelo menos uma dimensão (FIELD; MILES; FIELD, 2012); este obteve o KMO de 0,79 no geral, atendendo o critério de Kaiser (1974) acima de 0,5. Complementarmente foi estimado o KMO individual: Q01=0,81; Q02=0,81; $\mathrm{Q} 03=$ 0,84; Q04 = 0,77; Q05 = 0,76 e Q06 = 0,83, indicando que a maioria tem um bom nível de adequabilidade dos dados.

Após verificar que os dados são adequados para realizar uma análise fatorial, a confirmação de uma única dimensão e sua composição foi almejada (etapa 3). Na figura

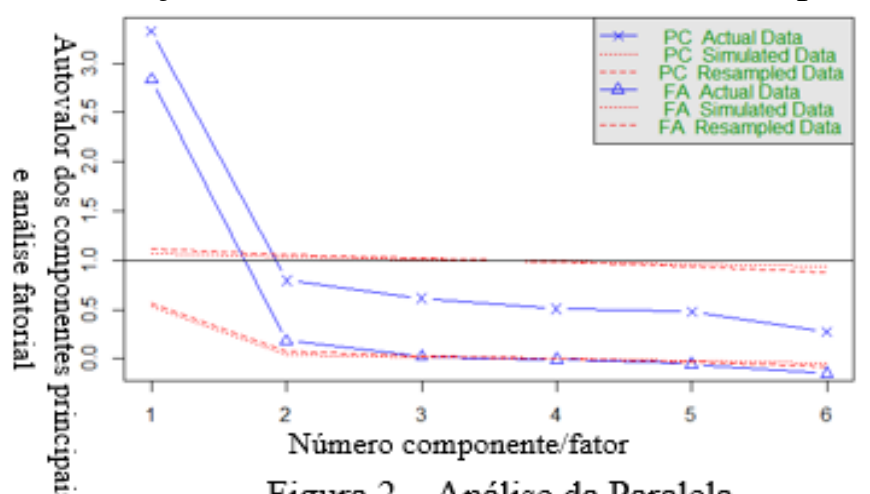

Figura 2 - Análise da Paralela

Fonte: Elaboração dos autores, 2021
2, há evidências de que os seis itens compõem uma única dimensão ou vice-e-versa, pois há um único fator tanto na análise dos componentes principais (o conjunto dos itens determina o construto) e quanto na análise fatorial (o construto determina o conjunto dos itens) acima de um, linha de Guttman-Kaiser, autovalor (eigenvalue) $>1$. O critério de scree de Cattell (1966)

também aponta para uma dimensão, pois a curva dos autovalores de ambos torna-se uma linha reta do primeiro até o segundo, ou sofre uma queda abrupta a partir do primeiro (cotovelo no segundo). O critério da análise paralela de Horn (1965) aponta para uma dimensão no $\mathrm{CP}$ e duas na $\mathrm{AF}$, acima da linha tanto dos simulados (simulated data) quanto a solução ótima da simulação (Resampled data). Em seguida, foram rotacionadas as cargas, a fim de observar se os itens são significantes para esta dimensão, e todas as cargas fatoriais atenderam o critério de Hair Junior et al (2005), pois foram maiores que $0,3(\mathrm{Q} 01=0.61 ; \mathrm{Q} 02=0.50 ; \mathrm{Q} 03=0.62 ; \mathrm{Q} 04=0.83 ; \mathrm{Q} 05=0.84$ e $\mathrm{Q} 06=0.64)$.

Em seguida foi analisada a consistência interna (etapa 4), a fim de verificar se o conjunto de itens mede de fato a dimensão do construto, clima de ambiente acadêmico. O KR foi 0,7, atendendo o critério ideal descrito no estudo de Kuder e Richardson (1937), significando que os itens em conjunto de fato medem a mesma dimensão. Na contribuição individual dos itens para a dimensão, correlação item-total (caso item for excluído do total), observa-se que, individualmente, os itens (Q01 = 0.43; $\mathrm{Q} 02=0.33 ; \mathrm{Q} 03=0.40$; $\mathrm{Q} 04=0.52 ; \mathrm{Q} 05=0.53$ e Q06 = 0.42) contribuem, significativamente, para a formação dessa dimensão, pois atenderam o critério de Streiner e Norman (2008), que é acima de 0,3 .

Estes resultados confirmam estatisticamente a presença do clima de ambiente acadêmico nos grupos do WhatsApp de sala de aula de graduandos. Em vista dessa confirmação, foram comparados os modelos, convergência dos parâmetros e ajustamento, a fim de obter o melhor modelo para mensurar a influência desse clima (etapa 5). A convergência dos parâmetros deu-se com aplicação do algoritmo EM (Esperança Maximização), o qual utiliza um processo iterativo para obter as aferições da máxima verossimilhança dos parâmetros em função da variável latente (ANDRADE, TAVARES; VALLE, 2000). O EM no Pacote mirt 1.33.2 é default para até 3 fatores, como também V. $19 \mathrm{~N}^{\mathrm{o}} 2$, Dezembro, 2021 
otimizador de m-etapas $B F G S$, aceleração Ramsay, número de quadratura retangular 61 (1 fator) e o tipo de densidade latente Gaussian para estes modelos. A convergência é observada na tabela 1 .

Tabela 1 - Convergências dos parâmetros

\begin{tabular}{c|c|c|c}
\hline Modelos & Iteration & Log-likelihood & Max-Change \\
\hline 2PL & 16 & -8083.837 & 0.00007 \\
\hline 3PL & 19 & -8083.952 & 0.00009 \\
\hline 3PLu & 27 & -8083.299 & 0.00008 \\
\hline 4PL & 40 & -8083.242 & 0.00010 \\
\hline
\end{tabular}

Fonte: Elaboração dos autores, 2021.

Todos os parâmetros dos modelos convergiram condicionados a um fator. Cabe salientar, que convergência nessa circunstância é de até 500 iterações. Na tabela 2, foi gerada uma anova, com o propósito de saber qual o melhor modelo, comparando com 2PL $\left(H_{0}\right)$.

Tabela 2 - Anova

\begin{tabular}{c|c|c|c|c|c|c|c|c}
\hline Modelos & AIC & AICc & SABIC & HQ & BIC & $\boldsymbol{X}^{\mathbf{2}}$ & $\mathbf{d f}$ & $\mathbf{p}$ \\
\hline 2PL & $\mathbf{1 6 1 9 1 . 6 7}$ & 16191.81 & 16222.95 & 16216.92 & $\mathbf{1 6 2 6 1 . 0 8}$ & & & \\
\hline 3PL & 16203.90 & 16204.19 & 16250.82 & 16241.78 & 16308.01 & -0.228 & 6 & $\mathbf{1}$ \\
\hline 3PLu & 16202.60 & 16202.89 & 16249.51 & 16240.47 & 16306.70 & 1.077 & 6 & $\mathbf{0 . 9 8 3}$ \\
\hline 4PL & 16214.48 & 16214.99 & 16277.04 & 16264.98 & 16353.29 & 1.192 & 12 & $\mathbf{1}$ \\
\hline
\end{tabular}

Fonte: Elaboração dos autores, 2021.

O modelo de 2PL de Birnbaum (1968), atendeu os critérios de Andrade, Tavares e Valle (2000) e Chalmers (2012), apresentando o menor AIC e BIC entre os modelos. O $P>0,05$, o que significa que $H_{0}$ (modelo de 2PL ajusta-se bem aos dados) foi aceito, portanto o modelo de 2PL foi escolhido e o seu ajustamento foi analisado na tabela 3.

Tabela 3 - Ajustamento do modelo 2PL

\begin{tabular}{c|c|c|c|c|c|c|c|c}
\hline $\boldsymbol{M}^{\mathbf{2}}$ & $\boldsymbol{d} \boldsymbol{f}$ & $\mathbf{p}$ & RMSEA & RMSEA_5 & RMSEA_95 & SRMSR & TLI & CFI \\
\hline 48.37 & 9 & $2.181608 \mathrm{e}-07$ & 0.04 & 0.03 & 0.05 & 0.03 & 0.98 & 0.99 \\
\hline
\end{tabular}

Fonte: Elaboração dos autores, 2021.

Conforme o critério de HAIR JUNIOR et al (2005) e Hu e Bentler (1999) o modelo tem um ajuste muito bom no RMSEA, TLI e CFI, e um ajuste perfeito no SRMSR. Deste modo, foi possível mensurar a influência do clima de ambiente acadêmico nas atividades acadêmicas dos graduandos com a curva característica do teste e item.

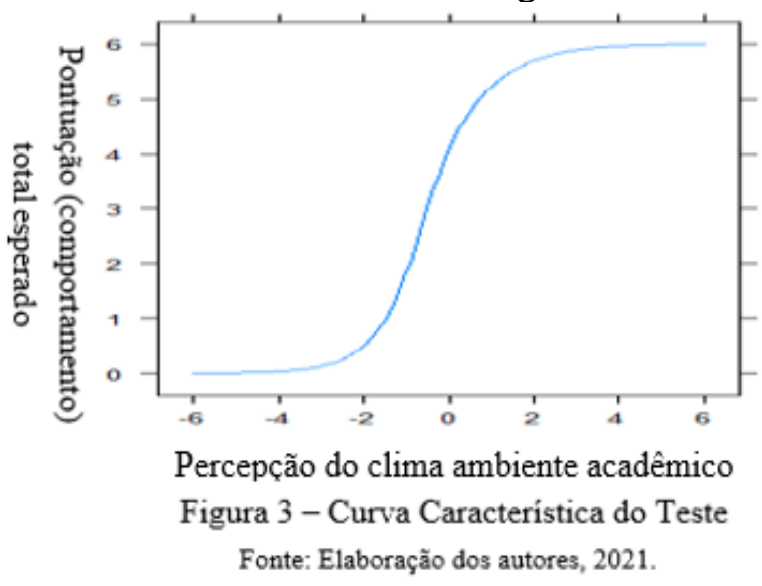

Fonte: Elaboração dos autores, 2021.

A curva característica do teste é observada na figura 3: as coordenadas na curva representam os números de comportamentos dos graduandos nas atividades acadêmicas nos grupos, condicionados ao seu nível de percepção desse clima. É possível diagnosticar que, graduandos com traço latente menor do que -2 , praticamente não são influenciados em seus comportamentos, nos seis itens. Também é observado que, entre -2 e 2, a inclinação na curva é mais íngreme. Isso significa que uma variação 
sutil na percepção do clima proporciona mudanças, significativas, nos comportamentos em aderir aos seis itens, essa variação pode ser incentiva ou não pelos envolvidos. Essa figura também traz à tona um desnivelamento no grupo, pois há graduandos que estão potencializando as atividades acadêmicas no grupo, enquanto outros não, o que é algo para trazer reflexões aos envolvidos sobre os efeitos disso ao longo prazo. Essa curva pode ser desmembrada para cada item, conforme a figura 4.

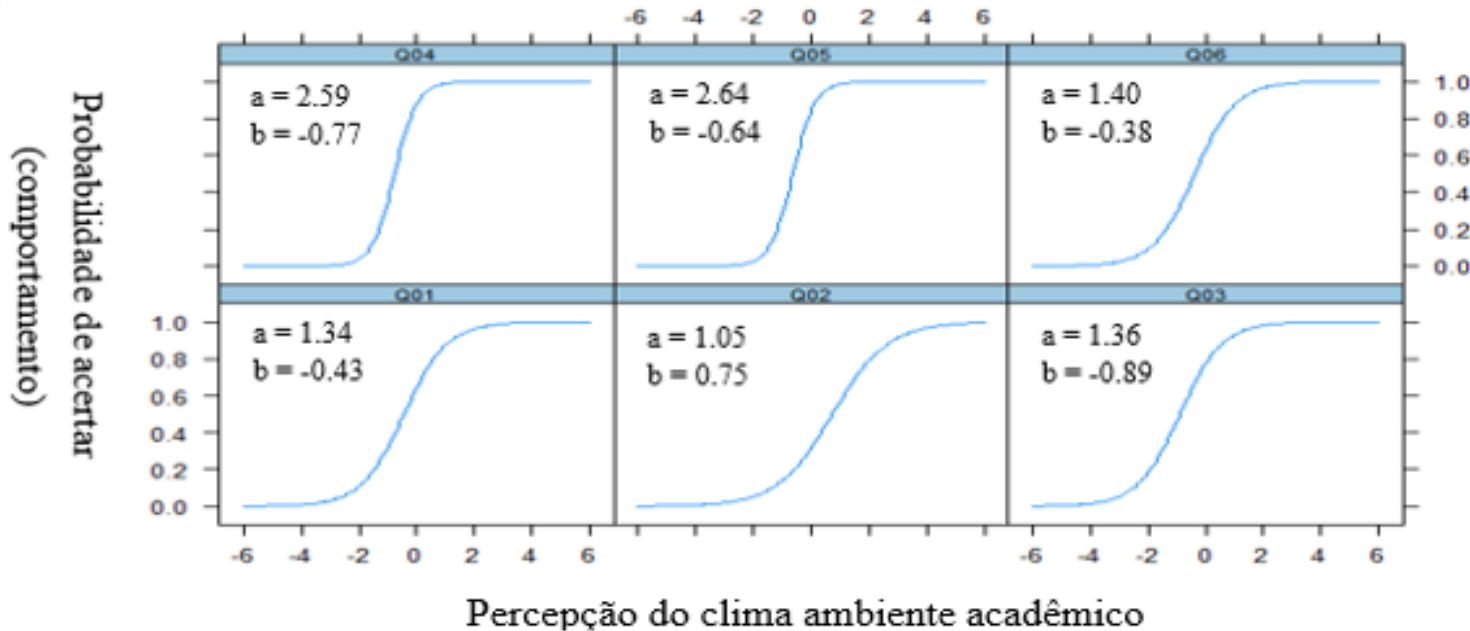

Figura 4 - Curva Característica do Item

Fonte: Elaboração dos autores, 2021.

As coordenadas na curva significam a probabilidade de acertar o item (manifestar o comportamento) condicionada ao nível de percepção do clima. $\mathrm{O}$ valor da dificuldade (item "b") é o ponto na curva que tem a probabilidade de $50 \%$ de acertar (manifestar o comportamento). Neste ponto, muda de concavidade na curva e tem maior inclinação, que é a discriminação (a), onde uma variação sutil na percepção do clima influencia mudanças significantes em aderir ou não ao comportamento no item, isto é, ele se torna mais propenso em ter ou não o comportamento.

É possível diagnosticar que todos os itens têm "b" no nível intermediário (dificuldade intermediária de manifestar o comportamento), mas o "a" com níveis diferentes (nível de influência) pelos critérios de Baker (2001) e Pasquali (2007), o Q01 (Já interagiu no grupo do WhatsApp da sala de aula, durante a aula, e que a maioria dos membros esteve presente na aula? Por exemplo, discutir os conteúdos, debater as matérias, dar avisos...) e Q02 (Já postou algum conteúdo que encontrou na internet relacionado com a aula, no grupo do WhatsApp da sala de aula, durante a aula? Por exemplo, indicações de materiais, confirmar algo que o professor disse na aula,...), possuem nível moderado do "a"; Q03 (Após a aula, já discutiu pelo grupo do WhatsApp da sala de aula os conteúdos estudados? Uma forma de fixar a matéria, trocar conhecimentos...) e Q06 (Na(s) atividade(s) extraclasse, através do grupo do WhatsApp da sala de aula, você já: passou Respostas? Uma das forma dos graduandos debaterem a solução, construírem o conhecimento em conjunto, pois nem sempre as respostas estão corretas, sendo necessário ir atrás da confirmação das respostas adquiridas), possuem o nível alto do "a"; Q04 (Antes da prova, você já discutiu pelo grupo do WhatsApp da sala de aula: dúvidas dos conteúdos? Uma forma de revisar a matéria, construir conhecimento...) e Q05 (Após a prova, você já discutiu pelo grupo do WhatsApp da sala de aula: o conteúdo da prova? Uma forma de confirmar aprendizagem, discutir o conteúdo...) possuem o nível perfeito do "a".

Essa figura, também traz à tona a comparação entre os itens, sendo que a curva mais à esquerda precisa de menos percepção para ter uma probabilidade maior de aderir o comportamento, ou seja, mais fácil, (Q04 e Q05 têm curva mais à esquerda). Além disso, V. $19 \mathrm{~N}^{\circ}$ 2, Dezembro, 2021

DOI: https://doi.org/10.22456/1679-1916.121356 RENOTE 
a comparação de todos os aspectos dos itens servem de subsídio para os envolvidos da educação discutirem esse fenômeno.

Esses resultados, confirmaram a presença do clima ambiente acadêmico nos grupos do WhatsApp de sala de aula e a sua influência nos comportamentos. Também foi possível identificar o nível de percepção do clima dos graduandos e seus efeitos nos comportamentos nas atividades acadêmicas (os seis itens do questionários). Essa informação é necessária para gerar reflexões e discussões sobre a importância desse clima no grupo do WhatsApp para educação.

\section{CONCLUSÃO}

O desenvolvimento do presente estudo possibilitou confirmar que o clima de ambiente acadêmico, também está presente nos grupos do WhatsApp de sala de aula de sala de aula de graduandos, e também possibilitou mensurar a influência nas atividades acadêmicas com a curva característica do teste e item.

Neste sentido, as informações geradas contribuem para reflexões e discussões dos envolvidos na educação, pois há diferenças nos níveis de percepção dos graduandos nos grupos, por consequência há um desnivelamento nos comportamentos sobre atividades acadêmicas no grupo, o que pode, a longo prazo, trazer algum tipo de efeito. Para estudos futuros, recomenda-se medir o clima constantemente ou compará-lo entre gêneros, idades, tipos de instituição, áreas de estudos, turnos e entre outros.

\section{REFERÊNCIAS}

ANDRADE, D.F; TAVARES, H.R.; VALLE, R.C. Teoria de Resposta ao Item: Conceitos e Aplicações. São Paulo: ABE - Associação Brasileira de Estatística, 2000.

BAKER, Frank. The Basics of Item Response Theory. Annapolis: University of Maryland, 2001.

BIRNBAUM, A. Some Latent Trait Models and Their Use in Inferring an Examinee's Ability. In: LORD, F.M.; NOVICK, M.R. (Ed.). Statistical theories of mental test scores. Reading: Addison-Wesley, 1968.

BRITO, Márcia de Sousa Terra; COSTA, Marcio da. Práticas e Percepções Docentes e Suas Relações com o Prestígio e Clima Escolar das Escolas Públicas do Município do Rio de Janeiro. Revista Brasileira de Educação, [S.L.], v. 15, n. 45, p. 500-510, dez. 2010. FapUNIFESP (SciELO). http://dx.doi.org/10.1590/s141324782010000300008.

CAI, L; HANSEN, M. Limited-Information Goodness-Of-Fit Testing of Hierarchical Item Factor Models. British Journal of Mathematical and Statistical Psychology, 66, 245-276, 2013.

CATTELL, Raymond B.. The Scree Test For The Number Of Factors. Multivariate Behavioral Research, [S.L.], v. 1, n. 2, p. 245-276, abr. 1966. Informa UK Limited. http://dx.doi.org/10.1207/s15327906mbr0102_10.

CHALMERS, R. Philip. Mirt: A Multidimensional Item Response Theory Package For The Environment. Journal of Statistical Software, [S.L.], v. 48, n. 6, p. 1-29, maio 2012. Foundation for Open Access Statistic. http://dx.doi.org/10.18637/jss.v048.i06.

FIELD, A.; MILES, J.; FIELD, Z. Discovering Statistics Using R. Sage Publications, 2012. $957 \mathrm{p}$. 
GUTTMAN, Louis. Some necessary conditions for common-factor analysis. Psychometrika, [S.L.], v. 19, n. 2, p. 149-161, jun. 1954. Springer Science and Business Media LLC. http://dx.doi.org/10.1007/bf02289162.

HAIR JUNIOR, J. F. et al. Análise multivariada de dados. 5. ed. Porto Alegre: Bookman, 2005. 593 p.

HORN, J. L. A Rationale and Test for the Number of Factors in Factor Analysis. Psychometrica, 30(2), 179-185, 1965.

HU, Li-Tze; BENTLER, Peter M. Cutoff Criteria For Fit Indexes in Covariance Structure Analysis: Conventional Criteria Versus New Alternatives. Structural Equation Modeling: A Multidisciplinary Journal, [S.L.], v. 6, n. 1, p. 1-55, jan. 1999. Informa UK Limited. http://dx.doi.org/10.1080/10705519909540118.

KAISER, Henry F.. The Application of Electronic Computers to Factor Analysis. Educational And Psychological Measurement, [S.L.], v. 20, n. 1, p. 141-151, abr. 1960. SAGE Publications. http://dx.doi.org/10.1177/001316446002000116.

KUDER, G. F; RICHARDSON, M. W. The Theory of The Estimation of Test Reliability. Psychometrika, v. 2, n. 3, September, p. 151-160. 1937.

LOPES, Cristiano G.; VAS, Braz B. O WhatsApp Como Extensão da Sala de Aula: O Ensino de História na Palma da Mão. Revista História Hoje, [S.L.], v. 5, n. 10, p. 159-179, 24 nov. 2016. Revista História Hoje. http://dx.doi.org/10.20949/rhhj.v5i10.291.

MAYDEU-OLIVARES, Albert; JOE, Harry. Limited Information Goodness-of-fit Testing in Multidimensional Contingency Tables. Psychometrika, [S.L.], v. 71, n. 4, p. 713-732, 11 nov. 2006. Springer Science and Business Media LLC. http://dx.doi.org/10.1007/s11336-005-1295-9.

MAYDEU-OLIVARES, Albert; HERNÁNDEZ, Adolfo; MCDONALD, Roderick P.. A Multidimensional Ideal Point Item Response Theory Model for Binary Data. Multivariate Behavioral Research, v. 41, n. 4, p. 445-472, dez. 2006. Informa UK Limited. http://dx.doi.org/10.1207/s15327906mbr4104 2.

MORO, Adriano et al. Avaliação do Clima Escolar por Estudantes e Professores: Construção e Validação de Instrumentos de Medida. Revista de Educação Pública, Cuiabá, v. 27, n. 64, p. 67-90, 29 dez. 2017. Revista de Educacão Pública. http://dx.doi.org/10.29286/rep.v27i64.3733.

SCHWEIG, Jonathan; HAMILTON, Laura S.; BAKER, Garrett. School and Classroom Climate Measures Considerations for Use by State and Local Education Leaders. 2009. Disponível em: https://www.rand.org/pubs/research_reports/RR4259.html. Acesso em: 13 fev. 2021. https://doi.org/10.7249/RR4259.

STREINER, David L; NORMAN, Geoffrey R. Health Measurement Scales: a practical guide to their development and use. 4. ed. Uk: Oxford University Press, 2008. 452 p.

PASQUALI, L. TRI - Teoria de Resposta ao Item: Teoria, Procedimentos e Aplicações.1. ed. Brasília: Laboratório de Pesquisa em Avaliação e Medida LabPAM, v. 1, 2007.

PASQUALI, Luiz; PRIMI, Ricardo. Fundamentos da Teoria da Resposta ao Item: TRI. Aval. psicol., Porto Alegre, v. 2, n. 2, p. 99-110, dez. 2003. Disponível em $<$ http://pepsic.bvsalud.org/scielo.php?script=sci arttext\&pid=S16770471200300020 $0002 \& \operatorname{lng}=$ pt\&nrm=iso $>$. Acessos em 15 fev. 2021.

V. $19 \mathrm{~N}^{\circ} 2$, Dezembro, 2021

DOI: https://doi.org/10.22456/1679-1916.121356 RENOTE 